\title{
Sex-dependent meiotic behaviour of B chromosomes in the grasshopper Eyprepocnemis plorans
}

\author{
N. Henriques-Gil,* \\ M. I. Canot and \\ J. L. Santos $†$
}

\author{
* Laboratorio de Genética, Colegio Universitario San \\ Pablo, C.E.U., Boadilla del Monte, Madrid 28660, \\ Spain. \\ † Departamento de Genética, Facultad de Biologia, \\ Universidad Complutense, Madrid 28040, Spain.
}

The meiotic behaviour of $\mathbf{B}$ chromosomes differs strongly between males and females of Eyprepocnemis plorans. In anaphase I of oogenesis the Bs always divide equationally though this segregation may become more or less aborted and a number of different secondary oocytes can be formed. In contrast, equational divisions of accessories in male meiosis are rare. This peculiar behaviour of $B$ chromosomes in the females does not seem to be associated with accumulation or loss.

\section{INTRODUCTION}

B chromosomes differ from the members of the normal karyotype in several features but specially because they may be present in different numbers, with an absence of clear effects on the carrier individual, and because they display nonmendelian modes of transmission. The peculiar modes of inheritance are in part a consequence of atypic behaviour during the meiotic divisions or, in the case of many plant species, in the postmeiotic mitoses (for review see Jones and Rees, 1982).

The fact that B chromosomes may be present in different numbers leads to a great range of possible configurations in the first meiotic division and, even when a pair of Bs exist in a cell, bivalents are not always formed. These phenomena result in many different patterns of inheritance.

The atypical behaviour of accessories is, in many cases, clearly associated with mechanisms of accumulation that tend to increase the frequency of Bs in the population. This is particularly so in the female where only one of the products of meiosis will form a functional gamete. For instance, in the grasshoppers Myrmeleotettix maculatus (Hewitt, 1973), Melanoplus femur-rubrum (Lucov and Nur, 1973) and Heteracris littoralis (Cano and Santos, 1988) Bs undergo a process of meiotic drive that increases the probability of inclusion in the egg. In these cases, the behaviour of Bs is probably not qualitatively different in males and females. Hewitt (1976) concluded that meiotic drive in Myrmeleotettix females was a consequence of spindle asymmetry with the B chromosome orientating towards the egg pole more often. Likewise, Cano and Santos (1988) observed in $H$. littoralis that in anaphase I cells one of the poles was more condensed than the other suggesting an asymmetric spindle in this species also.

Recent advances in the technique for the analysis of female meiosis after metaphase $I$ in grasshoppers (Henriques-Gil et al., 1986) have allowed us to contrast $\mathrm{B}$ chromosome behaviour between male and female meiosis in the grasshopper Eyprepocnemis plorans and our findings are described in this report.

\section{MATERIAL AND METHODS}

Male and female E. plorans were collected during September 1985 near the Spanish localities of Fuengirola (Málaga), Salobreña (Granada) and Daimuz (Valencia). The males were vivisected and the testes fixed in acetic-ethanol $1: 3$. The preparations of fixed material were made by the traditional squash technique with acetic-orcein staining.

The females were maintained in laboratory conditions in order to obtain mature eggs for meiotic observations. Female meiosis was analysed following the technique of Perry and Jones (1974) as modified by Henriques-Gil et al (1986). The number of B-carrying females from each popula- 
Table 1 Number of females analysed from each population and the number of cells scored for each stage of meiosis

\begin{tabular}{lrrrrrrr}
\hline Population & $\begin{array}{c}\text { No. of } \\
\text { fermales }\end{array}$ & \multicolumn{1}{c}{ Ml of cells } & AI & TI & MII & AII & TII \\
\cline { 3 - 9 } Daimuz & 10 & 11 & 10 & 16 & 10 & 5 & 8 \\
Salobreña & 8 & 8 & 12 & 10 & 8 & 9 & 6 \\
Fuengirola & 12 & 11 & 14 & 8 & 6 & 10 & 7 \\
\hline Total & 30 & 30 & 36 & 34 & 24 & 24 & 21 \\
\hline
\end{tabular}

tion and the number of cells analysed are given in table 1.

\section{RESULTS}

The natural populations analysed here were selected because of their B chromosome differences. Thus, in Daimuz accessory chromosomes are of the $B_{1}$ type, in Salobreña $B_{2}$ is usually found and in Fuengirola most accessories are of $B_{5}$ type. Other rare variants are also occasionally found in all three populations. The $\mathrm{B}$ chromosomes can be easily distinguished after $\mathrm{C}$-banding staining having an obvious monophyletic origin (HenriquesGil et al., 1984a).

These three types of B chromosomes show similar cytological behaviour in male meiosis (Henriques-Gil et al., 1982, 1984a). After an heteropycnotic state in the first meiotic prophase, at metaphase I univalent B chromosomes are usually placed outside of the equatorial plate (like the $\mathrm{X}$ chromosome; see fig. 1). If two Bs are present in the same individual, bivalents can be formed with variable frequencies showing an equatorial position similar to the autosomal bivalents. In anaphase $I$ the $B$ moves undivided towards one of the poles (fig. 3) giving rise to perfectly normal behaviour during the second division where each chromatid segregate to a pole. However, there is a low frequency of cells (less than 5 per cent) where orientation of univalent $\mathrm{Bs}$ in the equatorial plate is followed by equational segregation at anaphase I. Isolated chromatids in the second meiotic division are unable to move towards either pole and may become excluded from the rest of the chromosome complement forming a micronucleus.

The observations of female meiosis provide a quite different picture. Stages prior to the first metaphase could not be analysed due to the diffuse stage commonly occurring in grasshopper oocytes. At metaphase I the univalent B chromosomes orientate amphitelically in or near to, the equatorial plate (fig. 2).

In anaphase I the accessories divide in an equational manner in all cells analysed so far, and this applies for females from any of the natural populations sampled, presumably carrying, in each case, different types of $\mathrm{B}$ chromosomes. This can be clearly observed in figs 4 and 5 .

However, the late consequences of this phenomenon are not necessarily the same: in some cells the dividing univalent is placed near one of the anaphase poles suggesting that this division may not always be accomplished. This hypothesis is proved by the fact that the $\mathrm{B}$ chromosome constitution of secondary oocytes is variable. In addition to second division cells with one B-chromatid (the expected result after equational division), in a female having one B, different metaphase II cells may show zero chromatids or a two chromatid chromosome (see figs 6,7 and 8). Unfortunately, with our technique the number of cells available for analysis declines as meiosis progresses. Nevertheless, the limited quantitative study of the different types of second metaphases or their consequences in anaphase II indicates a random distribution of the $\mathrm{B}$ chromosome to the secondary oocyte: excluding the cells with $1 \mathrm{~B}$-chromatid, the number of secondary oocytes with one B chromosome and those without B is not statistically significant with respect to a $1: 1$ ratio (table 2 ).

\section{DISCUSSION}

It is well known that chromosome behaviour usually show some differences between male and female meiosis. For example, the rates of recombination are not the same for both sexes and there are some extreme cases like Drosophila males or Bombyx females with achiasmatic meiosis. In the species analysed here, the grasshopper E. plorans, a similar phenomenon was detected by Cano et al. (1987): the frequency of chiasmata is significantly lower in the females than in the males. Accordingly, we could also observe that the frequency of B-bivalents in two-B females is much lower than the frequencies commonly observed ir. two-B males (see Henriques-Gil et al., 1984b). (Later stages of meiosis could not be obtained in two-B females.)

The results reported here point out an active role of $\mathrm{B}$ chromosomes in determining their anaphase I behaviour depending on sex. The 100 per cent equational first anaphases in the females contrast with a very low frequency of this type of 

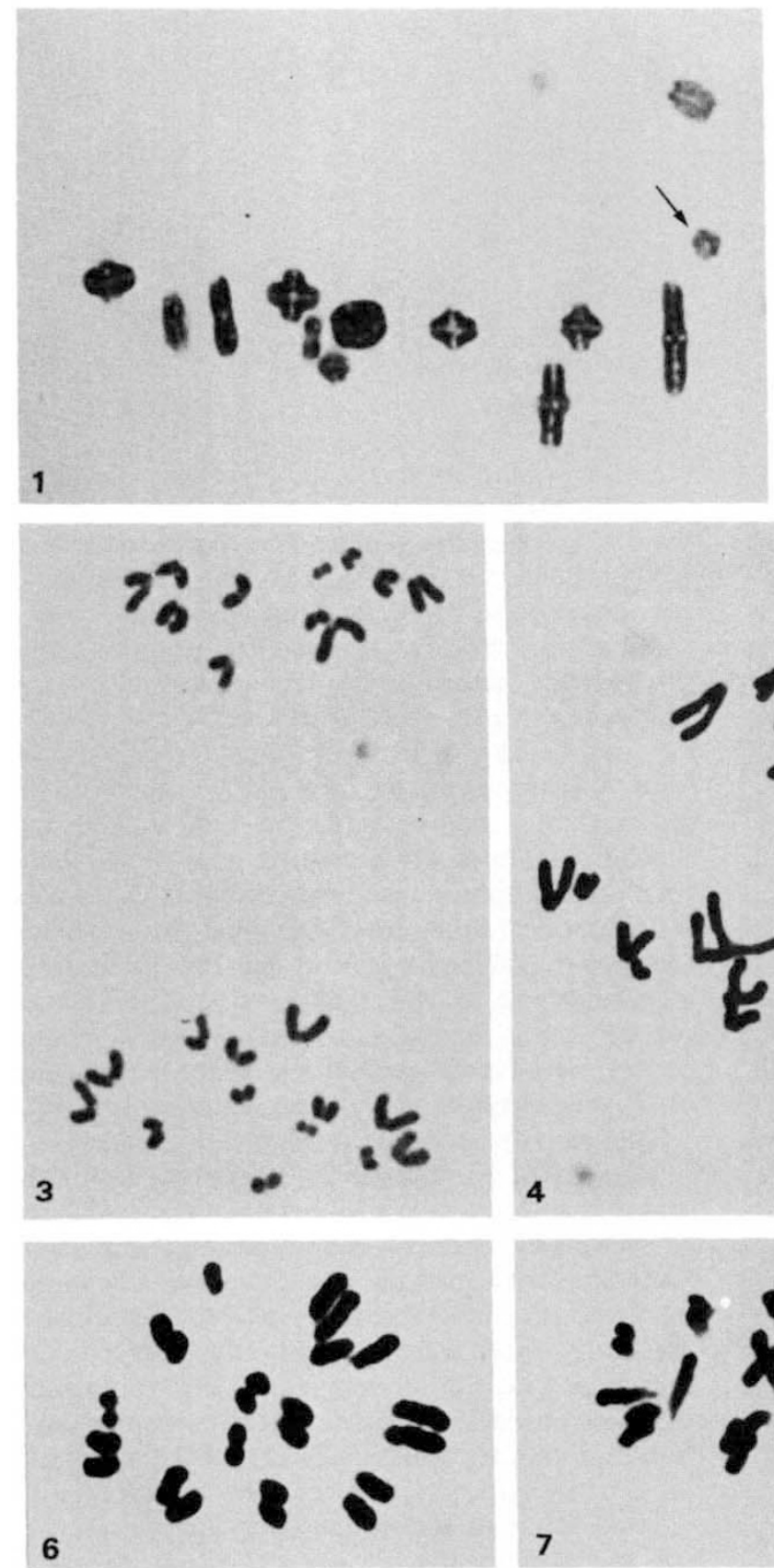
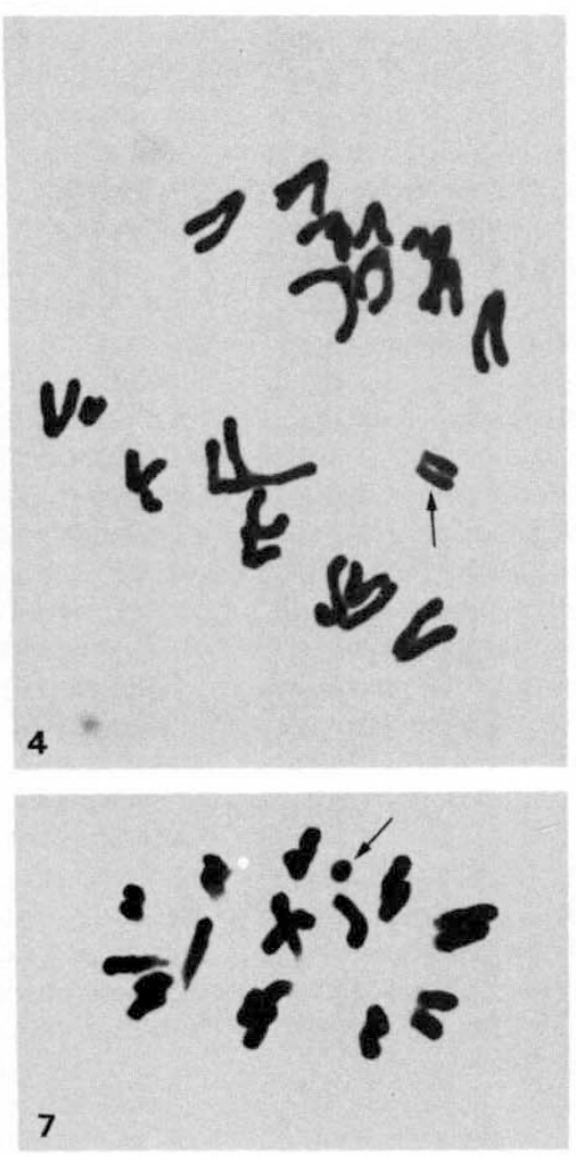
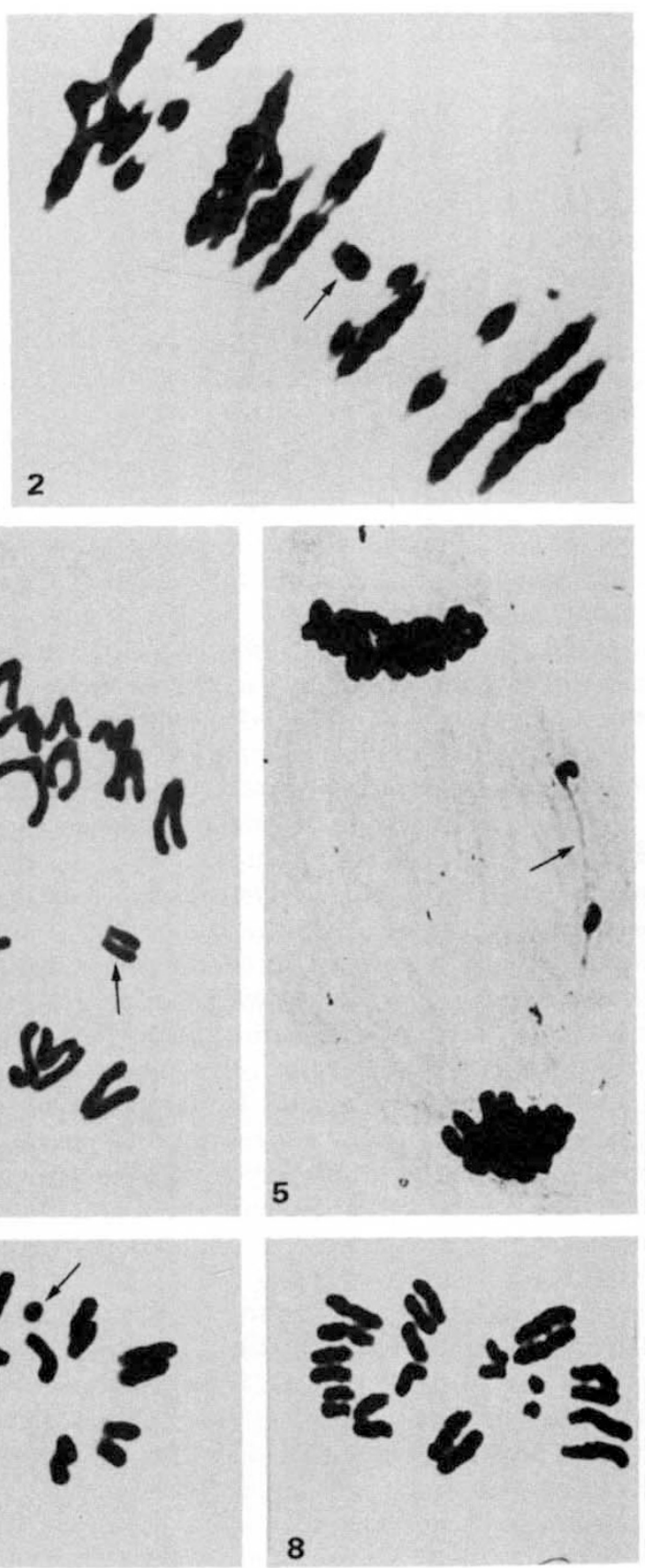

Figure 1. Metaphase I cell from a 1B male. The B chromosome is outside of the equatorial plate. In all cases, arrows indicate B chromosomes when they can be undoubtely identified.

Figure 2. Metaphase I cell from a $1 \mathrm{~B}$ female. The B is at the equatorial plate.

Figure 3. Anaphase I cell from a $1 \mathrm{~B}$ male, with 11 chromosomes in one pole and 13 in the other (11 autosomes plus the $\mathrm{X}$ and the B).

Figures 4-5. Anaphase I (4) and telophase I (5) cells from a $1 \mathrm{~B}$ female showing the B chromosome dividing in an equational manner.

Figures 6-8. Metaphase II cells from a 1B female containing 13 chromosomes, 12 chromosomes plus 1 B chromatid and 12 chromosomes respectively. 
Table 2 The distribution of B chromosomes in secondary oocytes of females of E. plorans having one $\mathrm{B}$ chromosome

\begin{tabular}{llcl}
\hline & \multicolumn{2}{l}{ Metaphase II and anaphase II cells } \\
\cline { 2 - 4 } Population & Without B & 1 B-chromosome & 1 B-chromatid \\
\hline Daimuz $\left(\mathrm{B}_{1}\right)$ & 7 & 8 & - \\
Salobreña $\left(\mathrm{B}_{2}\right)$ & 9 & 7 & 1 \\
Fuengirola $\left(\mathrm{B}_{3}\right)$ & 9 & 5 & 2 \\
\hline $\begin{array}{l}\text { Total } \\
\text { Expected after }\end{array}$ & 25 & 20 & 3 \\
random segregation & 22.5 & 22.5 & - \\
\hline
\end{tabular}

$\chi^{2}=0.556 ; 1 \mathrm{df} ; P>0.4$.

division in the males. Whatever mechanisms are involved, they do not affect the normal chromosomes which segregate identically in both sexes (except the X chromosome).

In $M$. maculatus and $H$. littoralis the behaviour of $\mathrm{B}$ chromosomes in male and female meiosis is similar but, in the latter, Bs tend to migrate towards the egg pole due to an asymmetry of the spindle which makes more probable the localization of the $B$ in the egg side than in the polar body side (Hewitt, 1976; Cano and Santos, 1988). The first meiotic division spindle of Eyprepocnemis may also be asymmetric since at late anaphase I and telophase 1 one pole is more condensed than the other (see figs 4 and 5), however the fact that Bs orientate in the equatorial plate indicates that there is no parallel with the cases of Myrmelotettix or Heteracris. In Eyprepocnemis a clear relationship between the capacity for equational division of $\mathrm{B}$ univalents and their position in the nucleus is observed (see Ostergren, 1951): if the B univalents are near to the metaphse I plate, they divide equationally whereas a polar disposition is followed by reductional division. In consequence, their behaviour must be probably determined when the plate is formed, that is, from diakinesis to metaphase I. Thus, either B-kinetochores have different properties in female than in male meiosis or a same B distinguishes some differences of the meiotic spindle of each sex.

It is important to note that in Eyprepocnemis some rare B variants are occasionally found (with a probable origin from the main ones) that show a high rate of equational segratation in anaphase I of males (Henriques-Gil et al., 1982, 1984a). This could therefore result from a loss in the mutant Bs of the ability to distinguish male from female meiosis exhibiting a high rate of equational segregations in male anaphase I. Although we have no reasons to suppose other kinds of behaviour, females carrying such derived types of B were not available for analysis at meiosis in order to observe if their behaviour is still the same or not. In maize, nondisjunction of $\mathrm{B}$ chromosomes in the second pollen grain mitosis also seems to be controlled by specific factors of the B chromosome located near the centromere (Carlson and Chou, 1981).

In a number of B chromosome systems studied in detail, atypical behaviour of accessories is often associated with accumulation mechanisms that probably counterbalance a decrease in frequency in natural populations produced by a lower fitness of B carriers (Jones and Rees, 1982). Accordingly, the most obvious meaning of the phenomena reported here could be to imply an accumulation of Bs in the female gametogenesis of Eyprepocnemis. However, observations do not seem to support this hypothesis. In first place, there is no evidence for such a mechanism because the frequency of Bs in second oocytes is not different to a $1: 1$ ratio of cells without $B$ and cells with one $B$, expected after a random segregation of the accessory in the first anaphase (the slight deviation is towards a lower number of Bs; see table 2). Secondly, and more importantly, Cano (1988) observed that the three types of B chromosomes analysed here are inherited in the offspring in a random manner with no tendencies to accumulation or loss from either sex. This argues against meiotic drive of $\mathrm{B}$ chromatids in anaphase II.

The significance of the behaviour described here is, therefore, far from clear. Hewitt (1974) suggested that after the origin of a B chromosome system it could become gradually more coadapted with the A-genome so as to produce a minimum of deleterious effects on the carrier individuals; in more neutral situations the rate of accumulation of Bs should also decrease avoiding an excessive increase in B frequency. Indeed, Blackman (1976) observed that in Euceraphis both males and 
females always have two B chromosomes. Both accessories pass to the functional spermatocytes but this apparent accumulation in compensated by some elimination, probably during oogenesis, to form the offspring again with a pair of B chromosomes. However, at this point, additional data are obviously needed in the case of Eyprepocnemis. Nevertheless, the fact that the different types of B chromosomes share the same peculiar behaviour during the female meiosis suggests strongly some importance in their maintenance.

\section{REFERENCES}

BLACKMAN, R. L. 1976. Cytogenetics of two species of Euceraphis (Homoptera, Aphididae). Chromosoma, 56, 393-408.

CANO, M. I. 1988. Estudio Citogenético de la Meiosis Femenina en Ortópteros. Tesis Doctoral, Universidad Complutense, Madrid

CANO, M. I., JONES, G. H. AND SANTOS, J. L. 1987. Sex differences in chiasma frequency and distribution in natural populations of Eyprepocnemis plorans containing B-chromosomes. Heredity, 59, 237-243.

CANO, M. I. AND SANTOS, J. L. 1988. The cytological basis of $\mathrm{B}$ chromosome accumulation in the grasshopper Heteracris littorialis (Ramb.). Heredity, 62, 91-95.

CARLSON, W. R. AND CHOU, T. S. 1981. B Chromosome nondisjunction in corn: control by factors near the centromere. Genetics, 97, 379-389.

HENRIQUES-GIL, N., SANTOS, J. L. AND GIRÁLDEZ, R. 1982. B-Chromosome polymorphism and interchromosomal chiasma interference in Eyprepocnemis plorans (Acrididae: Orthoptera). Chromosoma, 85, 349-359.
HENRIQUES-GIL, N., SANTOS, J. L. AND ARANA, P. 1984a. Evolution of a complex B chromosome polymorphism in the grasshopper Eyprepocnemis plorans. Chromosoma 89, 290-293.

HENRIQUES-GIL, N., ARANA, P. AND SANTOS, J. L. $1984 b$ Factors affecting the meiotic pairing of $\mathrm{B}$ chromosomes in the grasshopper Eyprepocnemis plorans. Can. J. Genet. Cytol., 26, 664-668.

HENRIQUES-GIL, N., JONES, G. H., CANO, M. I., SANTOS, J. L AND ARANA, P. 1986. Female meiosis during oocyte maturation in Eyprepocnemis plorans (Orthoptera: Acrididae). Can. J. Genet. Cytol., 28, 84-87.

HEWITT, G. M. 1973. Variable transmission rates of a B chromosome in Myrmeleottix maculatus (Thunb.) (Acrididae: Orthoptera). Chromosoma, 40, 83-106.

HEWITT, G. M. 1974. The integration of supernumerary chromosomes in the orthopteran genome. Cold Spring Harbor Symp. Quant. Biol., 38, 183-194.

HEWITT, G. M. 1976. Meiotic drive for B chromosomes in the primary oocytes of Myrmeleotetix maculatus (Orthoptera: Acrididae). Chromosoma, 56, 381-391.

JONES, R. N. 1975. B chromosome systems in flowering plants and animal species. Int. Rev. Cytol., 40, 1-100.

JONES, R. N. AND REES, H. 1982. B Chromosomes. Academic Press, London.

LUCOV, Z. AND NUR, U. 1973. Accumulation of B-chromosomes by preferential segregation in females of the grasshopper Melanoplus femur-rubrum. Chromosoma, 42, 289-306.

OSTERGREN, G. 1951. The mechanism of co-orientation in bivalents and mutlivalents. The theory of orientation of pulling. Hereditas, 37, 85-156.

PERRY, P. E. AND JONES, G. H. 1974. Male and female meiosis in Stethophyma grossum. Chromosoma, 47, 227-236. 